\title{
Differences in trigeminal and peripheral electrical pain perception in women with and without migraine
}

\author{
Barbara Gierse-Plogmeier · Reyhan Colak-Ekici · \\ Anne Wolowski · Ingrid Gralow · \\ Martin Marziniak $\cdot$ Stefan Evers
}

Received: 9 February 2009/ Accepted: 25 March 2009/Published online: 15 April 2009

(C) Springer-Verlag 2009

\begin{abstract}
Pain perception studies in migraine patients have shown trigeminal and peripheral pain facilitation during the migraine attack. We were interested in differences of trigeminal and peripheral pain perception between migraine patients during the migraine interval and healthy subjects. Perception of electrical pain stimulation was measured in 20 migraine subjects outside a migraine attack (10 migraine with aura and 10 migraine without aura) and in 20 healthy subjects. We recorded sensory and pain thresholds, pain ratings after suprathreshold stimulation, and pain rating after two trains of repetitive stimulation (i.e., pain facilitation). Migraine subjects showed a significantly higher pain rating after suprathreshold stimulation in the trigeminal region as compared to healthy subjects $(4.8 \pm 1.6$ versus $3.8 \pm 2.2, \mathrm{p}<0.04$ after Bonferroni correction) but not in the peripheral region. Furthermore, migraine subjects showed a pain facilitation after repetitive trigeminal stimulation whereas healthy subjects showed a pain habituation. We observed no significant differences between migraine subjects and healthy subjects for all parameters in the peripheral stimulation. Migraine patients with and without aura did not differ in any parameter. All subjects showed decreased sensory and pain thresholds
\end{abstract}

B. Gierse-Plogmeier · M. Marziniak · S. Evers $(\bowtie)$ Department of Neurology, University of Münster, Albert-Schweitzer-Str. 33, 48129 Münster, Germany e-mail: everss@uni-muenster.de

R. Colak-Ekici · A. Wolowski

Department of Prosthodontics, University of Münster, Münster, Germany

I. Gralow

Department of Anaesthesiology, University of Münster, Münster, Germany after trigeminal as compared to peripheral stimulation. Migraine subjects show an increased pain perception after trigeminal but not after peripheral pain stimulation as compared to healthy subjects. This phenomenon is probably due to the observed pain facilitation after painful trigeminal stimulation.

Keywords Migraine - Pain perception · Pain facilitation · Pain habituation · Pain thresholds

\section{Introduction}

The specific role of the trigeminovascular system for the pathophysiology of migraine has been increasingly recognised in the recent years. It has been shown that migraine patients exhibit a facilitated trigeminal pain perception in the attack as compared to healthy controls [1-3]. In particular, studies using laser-evoked potentials have been very useful to explore trigeminal nociception and habituation in migraine [4].

In an animal model of trigeminal pain, stimulation of the dura mater lead to decreased skin pain thresholds [5-7]. In experiments on humans, impaired skin sensibility in migraine patients has been shown when applying different stimuli such as cold (hand in ice water) [8] and heat [9] stimulation, mechanic pressure stimulation [3,9], intramuscular injection of inflammatory substances [10], or electrical stimulation of the skin $[9,11]$ or of the cornea [12]. In summary, an increased sensitivity of migraine patients as compared to healthy control subjects to these painful stimuli during a migraine attack has been shown [1, $2,4]$. The underlying mechanisms of this phenomenon are still unknown. It has been hypothesized that a peripheral and/or central sensitization is one of the main reasons for 
these decreased pain thresholds in migraine patients [3, 13-15].

However, the differences in peripheral pain perception between migraine patients and healthy subjects outside a migraine attack has received only little attention, and we are not aware of any study on the interictal pain thresholds in migraine patients as compared to healthy subjects. Therefore, we designed a study on sensory and pain thresholds in migraine patients outside an attack and compared the results to a control group. In addition, we were interested whether these possible differences can be related to trigeminal and/or peripheral pain perception and whether migraine features such as frequency or duration of the disease have an influence on this specific pain perception. Furthermore, we examined both single stimulation and repetitive stimulation as a measure of facilitation. Since it has been shown that the sex of both the experimenter and the subjects has a major impact on the subjective pain thresholds [16], we designed a study with exclusively women as experimenter and subjects.

\section{Methods}

\section{Subjects}

We enrolled 20 female subjects (mean age $25 \pm 5$ years) with a migraine according to the criteria of the International Headache Society [17], 10 of them without and 10 with aura (meaning that the majority of attacks occurred with an aura). All migraine and healthy subjects were diagnosed in a personal interview performed by an experienced headache expert. They were recruited from a tertiary headache center at the University of Münster, Germany. The control group comprised 20 female subjects (mean age $24 \pm 7$ years) without migraine and without any other idiopathic or present symptomatic headache. The mean duration of the migraine was $9.9 \pm 4.7$ years with a mean attack frequency of $1.2 \pm 2.6$ per month (range 0.2 to 5$)$.

Only women aged over 18 without any neurological or psychiatric disorder were considered. In particular, any depressive disturbance, regular intake of any drugs (except oral contraceptives), current smoking, and pregnancy were strict exclusion criteria. We included only women with an attack frequency of at least two migraine attacks per year for at least five years. At the time of experimental investigation, no migraine attack was present, and the previous migraine attack had to be at least three days ago. Also, we excluded subjects who had a migraine attack within two days after the procedure. Migraine attacks within two days before and during the menstrual bleeding were also excluded. The subjects were not informed about the hypothesis of our study (i.e., our expectation that migraine would influence interictal pain thresholds). We carefully registered the migraine history including frequency, age of onset, duration of migraine, occurrence of aura, and dates of the previous attacks. One week later, the subjects were contacted in order to register whether a migraine attack had occurred after the procedure.

The study was approved by the local ethics committee of the Faculty of Medicine, University of Münster. All subjects gave written informed consent.

\section{Procedure}

The migraine and the healthy subjects were investigated in a resting position. The pain stimulation was performed with a Dantec Keypoint device (Skovlunde, Denmark, 1997). We used wet stimulation electrodes placed with constant pressure in the first part of the examination on the skin over the tibial region and in the second part of the examination on the skin over the trigeminal region.

The sensory threshold was determined by application of single electric stimuli with increasing intensity until the stimulus was experienced. We started with $0.1 \mathrm{~mA}$ and then increased by steps of $0.1 \mathrm{~mA}$ until an intensity of $2.0 \mathrm{~mA}$. The further increase of stimulus intensity was done by steps of $0.2 \mathrm{~mA}$ until an intensity of $30 \mathrm{~mA}$ and after that level in steps by $0.5 \mathrm{~mA}$. The stimulus duration was $1 \mathrm{~ms}$.

After determination of the sensory threshold, we increased the stimulus intensity until the subjects rated the stimulus as painful for the first time. This pain threshold was also used in following assessments. After determination of the pain threshold, we applied a single stimulus with threefold pain threshold intensity. The subjects were asked to rate this stimulus on a visual analogue scale (VAS) between 0 and $100 \mathrm{~mm}$ [18]. This procedure was repeated three times, the arithmetic mean was considered as the pain intensity. Then, we applied 10 repetitive electric stimuli (first train) with a duration of $1 \mathrm{~ms}$, a frequency of $0.5 \mathrm{~Hz}$, and with an 1.5-fold pain threshold intensity. The subjects were then asked to rate the last stumulus of this train on the VAS. After $30 \mathrm{~s}$, we repeated this procedure of repetitive stimulation (second train) in order to determine pain facilitation or habituation. The subjects were neither informed about the relative nor about the absolute stimulus intensity.

The first part of the whole investigational procedure was performed over the tibial (i.e., peripheral) region. The stimulation was applied $10 \mathrm{~cm}$ under the insertation of the patella, the pressure of the stimulation electrode was mild and constant in order to stimulate primarily cutaneous sensory fibres. The second part of the investigational procedure was performed over the masseter (i.e. trigeminal) 
region with exactly the same protocol. The stimulation electrode was placed over the masseter muscle $2 \mathrm{~cm}$ over the horizontal mandibula. There was a break of 30 minutes between the two measurements to ensure that there was no order effect of the stimulation.

\section{Statistics}

Data are presented as arithmetic mean and standard deviation or as percentage. We used non-parametric testing (Wilcoxon-test, Friedman-test, Mann-Whitney-U-test, Spearman-rank-correlation coefficient). Since we applied multiple testing, $\mathrm{p}$ values are given after Bonferroni correction. Significance level was set at $\mathrm{p}=0.05$.

\section{Results}

We received complete data from all 20 migraine subjects and from all 20 healthy subjects.

Comparison between migraine and healthy controls

In Table 1, the results of the pain measurements are presented. There were two significant differences between migraine subjects and healthy subjects. First, the pain intensity of a given stimulus after trigeminal stimulation was rated significantly higher in migraine subjects than in healthy subjects. Second, the difference between the two trains of repetitive stimulation was significantly different for trigeminal stimulation. It was negative in healthy subjects pointing to a pain habituation. In migraine subjects, the difference was positive pointing to a pain facilitation or dishabituation rather than a normal pain habituation.

With respect to the migraine features, we found a significant correlation between the attack frequency and the difference between the two trains of repetitive stimulation (i.e. the amount of pain facilitation) in the trigeminal stimulation. The higher the attack frequency was the more negative the difference between the ratings of 2. train minus 1. train was $(r=-0.49 ; p=0.037)$. We found, however, no significant correlation between the duration of migraine in years and the pain facilitation $(\mathrm{r}=-0.22$; $\mathrm{p}=0.139$ ).

No differences were found with respect to pure sensory and pain thresholds between the two groups neither for the peripheral nor for the trigeminal stimulation. Further, we observed no significant difference in any parameter between migraine patients with and without aura (data not shown).

Comparison between trigeminal and peripheral nociception

In the two examination groups, the thresholds for pain perception were lower for the trigeminal as compared to the peripheral stimulation (Tables 2 and 3). The sensory thresholds did not show a significant difference between peripheral and trigeminal stimulation for both groups. However, the pain thresholds were significantly lower for

Table 1 Migraine data and results of pain measurement in healthy controls and in migraine subjects presented as arithmetic mean and standard deviation; statistical comparison by Mann-Whitney-U-test with subsequent Bonferroni correction

\begin{tabular}{llcc}
\hline & & Control $(\mathrm{n}=20)$ & Migraine $(\mathrm{n}=20)$ \\
\hline Migraine duration (years) & - & $9.9 \pm 4.7$ & $1.2 \pm 2.2$ \\
Migraine frequency per month & & & \\
Peripheral (tibial) region stimulation & $1.2 \pm 0.6$ & $1.1 \pm 0.6$ & $\mathrm{~ns}(\mathrm{p}=0.60)$ \\
Sensory threshold (mA) & $7.0 \pm 5.0$ & $6.7 \pm 4.8$ & $\mathrm{~ns}(\mathrm{p}=0.70)$ \\
Pain threshold (mA) & $2.6 \pm 1.8$ & $2.9 \pm 1.8$ & $\mathrm{~ns}(\mathrm{p}=0.76)$ \\
Threefold pain threshold stimulation (VAS in mm) & $2.9 \pm 1.9$ & $2.8 \pm 1.8$ & $\mathrm{~ns}(\mathrm{p}=0.99)$ \\
1. Train with 1.5-fold pain threshold stimulation (VAS in mm) & $3.0 \pm 2.2$ & $3.0 \pm 1.6$ & $\mathrm{~ns}(\mathrm{p}=0.68)$ \\
2. Train with 1.5-fold pain threshold stimulation (VAS in mm) & $0.1 \pm 0.8$ & $0.2 \pm 1.0$ & $\mathrm{~ns}(\mathrm{p}=0.76)$ \\
Difference between 2. train and 1. train & & & $\mathrm{ns}(\mathrm{p}=0.76)$ \\
Trigeminal region stimulation & $1.0 \pm 0.5$ & $1.0 \pm 0.3$ & $\mathrm{~ns}(\mathrm{p}=0.41)$ \\
Sensory threshold (mA) & $3.6 \pm 1.8$ & $3.3 \pm 2.0$ & $\mathrm{p}=0.04 *$ \\
Pain threshold (mA) & $3.8 \pm 2.2$ & $4.8 \pm 1.6$ & $\mathrm{~ns}(\mathrm{p}=0.29)$ \\
Threefold pain threshold stimulation (VAS in mm) & $3.9 \pm 2.3$ & $4.1 \pm 1.4$ & $\mathrm{~ns}(\mathrm{p}=0.33)$ \\
1. Train with 1.5-fold pain threshold stimulation (VAS in mm) & $3.8 \pm 2.4$ & $4.3 \pm 1.7$ & $\mathrm{p}=0.046^{*}$ \\
2. Train with 1.5-fold pain threshold stimulation (VAS in mm) & $-0.05 \pm 1.2$ & $0.2 \pm 1.3$ & \\
Difference between 2. train and 1. train & &
\end{tabular}

$* \mathrm{p}=0.12$ before correction 
Table 2 Comparison between the trigeminal and the peripheral pain measurement in migraine subjects

\begin{tabular}{lccc}
\hline & Trigeminal & Peripheral & Significance \\
\hline Sensory threshold (mA) & $1.0 \pm 0.3$ & $1.1 \pm 0.6$ & $\mathrm{p}=0.391$ \\
Pain threshold (mA) & $3.3 \pm 2.0$ & $6.7 \pm 4.8$ & $\mathrm{p}<0.001$ \\
Threefold pain threshold stimulation (VAS in mm) & $4.8 \pm 1.6$ & $2.9 \pm 1.8$ & $\mathrm{p}<0.001$ \\
1. Train with 1.5-fold pain threshold stimulation (VAS in mm) & $4.1 \pm 1.4$ & $2.8 \pm 1.8$ & $\mathrm{p}=0.002$ \\
2. Train with 1.5-fold pain threshold stimulation (VAS in mm) & $4.3 \pm 1.7$ & $3.0 \pm 1.6$ & $\mathrm{p}<0.001$ \\
Difference between 2. train and 1. train & $0.2 \pm 1.3$ & $0.2 \pm 1.0$ & $\mathrm{p}=0.641$ \\
\hline
\end{tabular}

Table 3 Comparison between the trigeminal and the peripheral pain measurement in healthy control subjects

\begin{tabular}{lrrr}
\hline & Trigeminal & Peripheral & Significance \\
\hline Sensory threshold (mA) & $1.0 \pm 0.5$ & $1.2 \pm 0.6$ & $\mathrm{p}=0.357$ \\
Pain threshold (mA) & $3.6 \pm 1.8$ & $7.0 \pm 5.0$ & $\mathrm{p}<0.001$ \\
Threefold pain threshold stimulation (VAS in mm) & $3.8 \pm 2.2$ & $2.6 \pm 1.8$ & $\mathrm{p}=0.003$ \\
1. Train with 1.5-fold pain threshold stimulation (VAS in mm) & $3.9 \pm 2.3$ & $2.9 \pm 1.9$ & $\mathrm{p}=0.003$ \\
2. Train with 1.5-fold pain threshold stimulation (VAS in mm) & $3.8 \pm 2.4$ & $3.0 \pm 2.3$ & $\mathrm{p}=0.007$ \\
Difference between 2. train and 1. train & $-0.05 \pm 1.2$ & $0.1 \pm 0.8$ & $\mathrm{p}=0.421$ \\
\hline
\end{tabular}

the trigeminal stimulation; and after stimulation with the threefold intensity of the pain threshold, both groups showed higher pain ratings for the trigeminal as compared to the peripheral stimulation. This corresponds to the observation that the pain rating after repetitive stimulation was higher for the trigeminal region between the two groups for the different trains applied in our stimulation paradigm.

When analysing the differences between the two trains (train 2 minus train 1) between trigeminal and peripheral stimulation, we did not find a significant difference between the two groups. In healthy subjects, we observed a decrease of pain perception in the second train for the peripheral but not for the trigeminal stimulation (Table 3). This means that healthy control subjects show a more pronounced pain habituation for peripheral than for trigeminal stimulation whereas migraine subjects did not show a difference in habituation to painful stimuli between peripheral and trigeminal stimulation. However, these trends were not statistically significant.

\section{Discussion}

In this study, we observed relevant differences in the perception of painful electric stimuli between female migraine subjects and female healthy subjects. Since we measured only migraine attacks outside the perimenstrual days, we believe that the influences of the hormonal cycle are of minor importance.

First, we found increased pain thresholds during peripheral stimulation as compared to trigeminal stimulation both in healthy subjects and in migraine subjects. This confirms similar observations in previous studies [19]. One of the underlying mechanisms for this difference in thresholds might be that both groups show a higher density of nociceptors in the trigeminal region as compared to other peripheral regions of the body.

However, we detected a significant difference in pain facilitation after trigeminal stimulation. Female patients in the migraine interval are more sensitive to repetitive trigeminal stimuli than female healthy subjects. The first showing increased pain rating and the latter showing decreased pain rating after repetitive stimulation. It is still unknown what the underlying mechanims of this specific pain perception in migraine subjects is. As several authors also suggested, we assume that subjects with migraine have a central sensitisation with a different neuronal network activity in the brainstem [1-3, 7, 11-15]. A further supporting argument for this hypothesis is that the increased pain facilitation is correlated with the migraine attack frequency. This points to a central facilitation rather than to a peripheral such as changes of cutaneous nociception or of nerve fibres and has also been shown in a similar study [15]. However, in a recent study on the nociceptive blink reflex, the loss of habituation to painful stimuli in migraine subjects interictally was not higher in those subjects with high attack frequency [20]. This has been explained by genetic mechanisms rather than trigeminal pain sensitization. The difference from our study might be explained by the fact that we evaluated complex (i.e., stimulating different fibre systems) pain stimuli and not brainstem potentials as a response to mild C-fibre mediated stimuli which were not rated as painful by the subjects. We observed no significant difference in any parameter between migraine patients with and without aura suggesting that the liability to a migraine aura has no major impact on pain processing. 
Several migraine subjects show a facial allodynia during the migraine attack [1]. This phenomenon also supports the view that meningeal and other trigeminal painful stimuli are perceived by the same nociceptive network. This is another evidence for the different pain perception of trigeminal and peripheral stimuli.

Further, psychosomatic mechanisms might be of importance for the observed phenomenon. The repetitive facial and head pain in migraine patients during their regular attacks can lead to a facilitation of all painful trigeminal stimuli in these subjects. Chronic pain patients show a facilitation rather than a habituation to repetitive pain.

We observed some differences as compared to similar studies. Although we could reproduce the finding that pain facilitation is present in subjects with tension-type headache also for migraine subjects, this facilitation was only found in trigeminal pain perception but not in peripheral [19]. Furthermore, we could not replicate the general finding that peripheral pain thresholds are lower in headache patients as compared to healthy control subjects [13]. However, we could show that the trigeminal pain facilitation is also present in the migraine interval and not only during the migraine attack. This is in concordance to two previous studies [20, 21].

There are some limitations in our study. First, some of our subjects had difficulties in determining the pain threshold whereas the sensory threshold was easy to detect in all subjects. This could have caused a systematic bias. Second, although the experimental procedure was done by the same investigator in all cases, we cannot rule out fluctuations in the different manual applications of stimuli; in particular, we cannot be sure that we stimulated exactly the same fibre types in all subjects. Third, we did not completely control for the menstrual cycle which might be an independent factor influencing pain perception in general [22]. Finally, the relatively high interindividual variability in the thresholds and pain ratings make it, with respect to statistical analysis, difficult to detect systematic differences between groups of subjects. Furthermore, the stimulation was not blinded which could lead to a presentation of expected answers. However, it is very difficult to perform a blinding of stimulation in such a study design. Our data apply only to subjects with a long duration of migraine. It is unknown whether our findings are also applicable to subjects who had just started to have migraine attacks.

With our study design, we are not able to determine whether the differences in pain perception between migraine and healthy subjects is a primary or a secondary phenomenon. It has still to be elucidated whether the decreased pain thresholds in migraine subjects are due to the hereditary liability of a subject for migraine or whether this occurs during the history of migraine as a result of repetitive migraine attack perception. The detection of this mechanism would be highly interesting for the question how to develop specific migraine prophylactic agents. On the other hand, studying differences in pain thresholds between migraine subjects with and without prophylactic medication or before and after initiation of prophylactic medication would also elucidate this point.

We conclude that our method of measuring pain thresholds and pain facilitation is very robust with respect to intraindividual reproducibility and application in pain research. However, the limitation of this method of electrical painful stimuli is that we cannot exactly differentiate which nerve fibres are involved in the pain conduction. Electrical painful stimuli are primarily processed by $\mathrm{A} \beta$ fibres. They are also processed by $\mathrm{A} \delta$-fibres and can also activate $\mathrm{C}$-fibres. Therefore, it has still to be determined whether the pain facilitation in migraine patients is processed by a specific subtype of nerve fibres or whether it is independent from the nerve fibre conduction.

\section{Conflict of interest None.}

\section{References}

1. Burstein R, Cutrer MF, Yarnitsky D (2000) The development of cutaneous allodynia during migraine attack clinical evidence for the sequential recruitment of spinal and supraspinal nociceptive neurons in migraine. Brain 123:1703-1709

2. Burstein R, Yarnitsky D, Goor-Aryeh I, Ransil BJ, Bajwa ZH (2000) An association between migraine and cutaneous allodynia. Ann Neurol 47:614-624

3. Burstein R (2001) Deconstructing migraine headache into peripheral and central sensitization. Pain 89:107-110

4. de Tommaso M (2008) Laser-evoked potentials in primary headaches and cranial neuralgias. Expert Rev Neurother 8:13391345

5. Strassman AM, Raymond SA, Burstein R (1996) Sensitization of meningeal sensory neurons and the origin of headaches. Nature 384:560-564

6. Burstein R, Yamamura H, Malick A, Strassman AM (1998) Chemical stimulation of the intracranial dura induces enhanced responses to facial stimulation in brain stem trigeminal neurons. $\mathrm{J}$ Neurophysiol 79:964-982

7. Yamamura H, Malick A, Chamberlin NL, Burstein R (1999) Cardiovascular and neuronal responses to head stimulation reflect central sensitization and cutaneous allodynia in a rat model of migraine. J Neurophysiol 81:479-493

8. Bishop KL, Holm JE, Borowiak DM, Wilson BA (2001) Perceptions of pain in women with headache: A laboratory investigation of the influence of pain-related anxiety and fear. Headache 41:494-499

9. Weissmann-Fogel I, Sprecher E, Granovsky Y, Yarnitsky D (2003) Repeated noxious stimulation of the skin enhances cutaneous pain perception of migraine patients in-between attacks: clinical evidence for continous sub-treshold increase in membrane excitability of central trigeminovascular neurons. Pain 104:693-700

10. Mørk H, Ashina M, Bendtsen L, Olesen J, Jensen R (2003) Induction of prolonged tenderness in patients with tension-type 
headache by means of a new experimental model of myofascial pain. European Journal of Neurology 10:249-256

11. Marlowe NI (1992) Pain sensitivity and headache: an examination of the central theory. J Psychosom Res 36:17-24

12. Sandrini G, Cecchini AP, Milanov I, Tassorelli C, Buzzi MG, Nappi G (2002) Electrophysiological evidence for trigeminal neuron sensitization in patients with migraine. Neurosci Lett 317:135-138

13. Malick A, Burstein R (2000) Peripheral and central sensitization during migraine. Funct Neurol 15(Suppl 3):28-35

14. Bendtsen L (2002) Sensitization: its role in primary headaches. Curr Opin Invest Drugs 3:449-453

15. Buchgreitz L, Lyngberg AC, Bendtsen L, Jensen R (2006) Frequency of headache is related to sensitization: A population study. Pain 123:19-27

16. Gijsbers K, Nicholson F (2005) Experimental pain tresholds influenced by sex of experimenter. Percept Mot Skills 101:803-807

17. Headache Classification Committee of the International Headache Society (2004) International Classification of Headache Disorders, 2nd edn. Cephalalgia 24(Suppl 1):1-160
18. Price DD, McGrath PA, Rafii A, Buckingham B (1983) The validation of visual analogue scales as ratio scale measures for chronic and experimental pain. Pain 17:45-56

19. Ashina S, Babenko L, Jensen R, Ashina M, Magerl W, Bendtsen L (2005) Increased muscular pain sensitivity in cephalic region but not in extremity in chronic tension-type headache. Eur $\mathbf{J}$ Neurol 12:543-549

20. Di Clemente L, Coppola G, Magis D, Fumal A, De Pasqua V, Di Piero V, Schoenen J (2007) Interictal habituation deficit of the nociceptive blink reflex: an endophenotypic marker for presymptomatic migraine? Brain 130:765-770

21. Kitaj MB, Klink M (2005) Pain thresholds in daily transformed migraine versus episodic migraine headache patients. Headache 45:992-998

22. Soderberg K, Sundstrom Poromaa I, Nyberg S, Backstrom T, Nordh E (2006) Psychophysically determined thresholds for thermal perception and pain perception in healthy women across the menstrual cycle. Clin J Pain 22:610-616 